\title{
Coupled exciton model with off-diagonal disorder for optical excitations in extended dendrimers
}

\author{
Kikuo Harigaya周 \\ Physical Science Division, Electrotechnical Laboratory, \\ Umezono 1-1-4, Tsukuba 305-8568, Japan币 \\ National Institute of Materials and Chemical Research, \\ Higashi 1-1, Tsukuba 305-8565, Japan \\ Kanazawa Institute of Technology, \\ Ohgigaoka 7-1, Nonoichi 921-8501, Japan \\ (Received \\ )
}

\begin{abstract}
A phenomenological coupled exciton model is proposed in order to characterize optical excitations in extended dendrimers. An onsite exciton state is assigned at each phenyl rings and a nearest neighbor hopping integral which obeys the Gaussian distribution is considered between the exciton states. The decreasing optical excitation energy with respect to the dendrimer size indicates the presence of exciton funnels along the backbone of the dendrimers. Therefore, the extended dendrimers can work as artificial fractal antenna systems which capture energy of light.
\end{abstract}

\footnotetext{
*E-mail address: harigaya@etl.go.jp; URL: http://www.etl.go.jp/ harigaya/

${ }^{\dagger}$ Corresponding address
} 
Recently, the dendrimer supermolecules with antenna structures have been investigated extensively. After capture of light at the outer edges of the molecule, generated excitons migrate along the legs of the molecular structures and carry energy obtained from light. Then, the excitons move to a certain core to localize there, or move to the center of the supermolecule in order to emit light by recombination of electrons and holes. Because energy of light is captured and the energy is transfered by excitons, the supermolecules might act as artificial molecular antenna. Therefore, the design of the molecular structures and their optical properties are quite attractive in view of scientific interests as well as their potential for technological applications.

One example of antenna supermolecules has dendrimeric structures. It is a family of molecules composed of phenyl rings and acetylene units, namely, extended dendrimers [1-5]. Their geometrical structures are illustrated in Fig. 1. Figure 1 (a) shows the diphenylacetylene with its abbreviated notation. Figure 1 (b) shows the family of extended dendrimers: D4, D10, D25, D58, and D127. The number in their names means the number of phenyl rings in the molecules. In the D4 and D10 dendrimers, each leg is composed of two single bonds and one triple bond. In D25, three central legs are made of one phenyl rings and two short legs (shown as Fig. 1 (a)). In D58, three central legs are composed of two phenyl rings and three short legs (Fig. 1 (a)). The next connecting legs via the phenyl vertex are like the central legs of D25. In D127, the three central legs are made of three phenyls and four short legs (Fig. 1 (a)). In this way, the extended dendrimers have fractal molecular structures, and the length of the central legs becomes longer as the size of the dendrimer becomes larger.

Optical experiments of the extended dendimers [3-5] show that the optical gap decreases as the dendrimer size becomes larger. The optical spectra have features which can be understood as contributions from legs of phenylacetylene oligomers. The energy of each feature agrees with that of oligomers. The excitation energy becomes smaller as the length of the legs becomes larger. Therefore, the presence of exciton migration pathways along the legs or the backbone of the supermolecules has been concluded.

Another example of the antenna supermolecules has structures with four zinc-containing 
tetraarylporphylins linked to a central, unmetallated porphylin through ethyne bonds [6]. They show migration characters of excitons, and are interesting as light harvesting antennas. Such alternative molecular designs, replacement of molecules at the vertexes, different structure of leg chains, and so on, have been investigated intensively. Although various molecular structures are of interests, we would like to concentrate upon optical excitation properties of the extended dendrimers in this paper because of the varieties of possible structures which will require much more theoretical efforts in future.

Theory of optical excitations in the dendrimers has not been reported so often, and for example energy transfer has been investigated by solving phenomenological probability process equations [7]. The rates of the excitation flow along the legs of the dendrimer have been introduced, and the mean passage time has been obtained theoretically.

In this paper, we would like to give rise to a new theoretical model composed of coupled exciton states with off-diagonal disorder. We note that the theoretical model of dipole moments [8] has been used in order to characterize optical excitations of the photosynthetic unit of purple bacteria, which is the biological analog of the dendrimeric supermolecule. In the present model, an onsite exciton state is assigned at each phenyl ring. There are two possibilities for the nearest neighbor interactions: (1) When the interactions occur by dipole-dipole couplings, the direction of the transition dipole moment is by no means parallel with the electric field of light and cannot be spatially correlated. Interaction strengths between neighboring dipole moments may vary among positions of dipole pairs. They can be looked as randomly distributed. Therefore, we assume that the nearest neighbor interactions obey the Gaussian distribution function. (2) The second candidate of the interaction between neighboring exciton states is an exciton flow characterized with the strengths $t_{e} t_{h} / \Delta_{\text {ex }}$ by perturbation, where $t_{e}$ and $t_{h}$ are hopping integrals of electrons and holes, $\Delta_{\mathrm{ex}}$ is the excitation energy of the electron hole pair. We assume that the mean value of the interaction is zero and the standard deviation of the interaction $J$ is one of theoretical parameters in the Gaussian distribution. Another theoretical parameter in the model is the site energy $E$ which specifies the central energy position of excitons in the optical 
spectra. The following is our tight binding model:

$$
H=E \sum_{i}|i\rangle\langle i|+\sum_{\langle i, j\rangle} J_{i, j}(|i\rangle\langle j|+\text { h.c. }),
$$

where $i$ means the $i$ th site of the phenyl ring, $|i\rangle$ is an exciton state at the site $i$, the sum with $\langle i, j\rangle$ is taken over neighboring pairs of sites, and the distribution of $J_{i, j}$ is determined by the Gaussian function,

$$
P\left(J_{i, j}\right)=\frac{1}{\sqrt{4 \pi} J} \exp \left[-\frac{1}{2}\left(\frac{J_{i, j}}{J}\right)^{2}\right] .
$$

Though detailed theoretical treatments are not the same altogether, the related exciton models have been used for the photosynthetic unit of purple bacteria [8] and the J-aggregate systems [9-11]. The diagonalization of eq. (1) gives energies of one exciton states measured from the energy of the ground state.

The model eq. (1) is diagonalized numerically for the five types of the extended dendrimers: D4, D10, D25, D58, and D127. The lowest eigenvalue always gives the energy position of the optical absorption edge because the state with the lowest energy is always allowed for dipole transition from the ground state. This is checked by looking at the parity of the wave function for each dendrimer. In TABLE I, we show the energy of the absorption edge as a function of the parameters $E$ and $J$. Here, the number of disorder samples is 10000, and this gives the well converged average value of the optical excitation energy.

Figure 2 shows one example of the comparison of the calculation with experiments for the parameters $E=37200 \mathrm{~cm}^{-1}$ and $J=3552 \mathrm{~cm}^{-1}$. We find fairly good agreement between the experiments and calculations. Two results have the trend that the lowest optical excitation energy decreases as the dendrimer size becomes larger. Therefore, it is clarified that the presence of exciton migration funnels is well described by the present coupled exciton model with offdiagonal disorder. The interaction strength $J$ is one order of magnitudes larger than that of the purple bacteria [8], indicating the stronger contact between neighboring exciton states. When the flow of excitons occur by hopping of electrons and holes, the interaction strength is characterized as $t_{e} t_{h} / \Delta_{\text {ex }}$ by perturbation. With assuming $t_{e} \sim t_{h} \sim 0.5 t$ and $\Delta_{\text {ex }} \sim 2 t$ 
where $t$ is the resonance integral of the $\pi$ orbitals of the phenyl ring, we obtain a characteristic magnitude: $|J| \sim 0.1 t$. Here, the values of $t_{e}$ and $t_{h}$ smaller than $t$ are assumed, because there are three bondings between the neighboring phenyls. We have used $\Delta_{\mathrm{ex}}=2 t$, as the energy difference between the highest occupied state and the lowest unoccupied state of a single phenyl is $2 t$. The quantity $|J| \sim 0.1 t$ is also of the same order of magnitudes with the above parameter $J=3552 \mathrm{~cm}^{-1}$, because $t \sim 2 \mathrm{eV} \sim 25000 \mathrm{~cm}^{-1}$. Thus, our theoretical parameter can characterize exciton flows along the legs of dendrimers very well.

The extended dendrimers are the rare example where $\pi$-conjugated electron systems are present along the acetylene based legs. In most of dendrimers (see the recent review [12] for example), the systems are composed of $\sigma$-bondings rather than $\pi$-bonds. Owing to the presence of exciton funnels composed of $\pi$-bonds, the extended dendrimers can work as an artificial fractal antenna which captures energy of light.

In the compact dendrimers (the another form of diphenylacetylene based dendrimers), the optical absorption edge less depends on the molecule size [3]. This might come from the very huge steric repulsions among neighboring legs, and therefore the mutual interactions between legs are hindered easily by geometric effects. The nearly constant absorption edge with respect to the dendrimer size indicates that values of the interaction strengths $J_{i, j}$ of the compact dendrimers are much smaller than those of the extended dendimers when the present coupled exciton model is applied to. Such the difference of the parameter values can characterize the different dependence on the system size of the extended and compact dendimers.

In summary, we have proposed the coupled exciton model with off-diagonal disorder in order to characterize optical excitations in the extended dendrimers. The decreasing optical excitation energy with respect to the dendrimer size indicates the presence of exciton pathways along the backbone of the dendrimers.

\section{Acknowledgements}


Useful discussion with the members of Condensed Matter Theory Group

(http://www.etl.go.jp/ ${ }^{\sim}$ theory/), Electrotechnical Laboratory is acknowledged. Numerical calculations have been performed on the DEC AlphaServer of Research Information Processing System Center (RIPS), Agency of Industrial Science and Technology (AIST), Japan. 


\section{References}

[1] Z. Xu and J. S. Moore, Acta Polym. 45, 83 (1994).

[2] C. Devadoss, P. Bharathi, and J. S. Moore, J. Am. Chem. Soc. 118, 9635 (1996).

[3] R. Kopelman, M. Shortreed, Z. Y. Shi, W. Tan, Z. Xu, J. S. Moore, A. Bar-Haim, and J. Klafter, Phys. Rev. Lett. 78, 1239 (1997).

[4] M. R. Shortreed, S. F. Swallen, Z. Y. Shi, W. Tan, Z. Xu, C. Devadoss, J. S. Moore, and R. Kopelman, J. Phys. Chem. 101, 6318 (1997).

[5] S. F. Swallen, Z. Y. Shi, W. Tan, Z. Xu, J. S. Moore, and R. Kopelman, J. Lumin. 76\&77, 193 (1998).

[6] R. W. Wagner, T. E. Johnson, and J. S. Lindsey, J. Am. Chem. Soc. 118, 11166 (1996).

[7] A. Bar-Haim and J. S. Klafter, J. Lumin. 76\&77, 197 (1998).

[8] T. Ritz, X. Hu, A. Damjanović, and K. Schulten, J. Lumin. 76\&77, 310 (1998).

[9] F. C. Spano, J. R. Kuklinski, and S. Mukamel, Phys. Rev. Lett. 65, 211 (1990).

[10] F. C. Spano, J. R. Kuklinski, and S. Mukamel, J. Chem. Phys. 94, 7534 (1991).

[11] T. Kato, F. Sasaki, S. Abe, and S. Kobayashi, Chem. Phys. 230, 209 (1998).

[12] "Dendrimers", ed. F. Vögtle (Springer, Berlin, 1998). 
TABLE I. The energy of the lowest optical excitation.

\begin{tabular}{lc}
\hline \hline Dendrimer & Absorption edge \\
\hline D4 & $E-1.6037 J$ \\
D10 & $E-2.3346 J$ \\
D25 & $E-2.8078 J$ \\
D58 & $E-3.1588 J$ \\
D127 & $E-3.4338 J$ \\
\hline \hline
\end{tabular}

\section{Figure Captions}

Fig. 1. (a) Diphenylacetylene and its abbreviated notation. The closed circle means a phenyl ring, and the connecting line means two single bonds and one triple bond. (b) The extended dendimers: D4, D10, D25, D58, and D127. The number in their names is number of phenyl rings in fractal supermolecules.

Fig. 2. Optical absorption edge of the five dendrimers: D4, D10, D25, D58, and D127. The crosses are taken from experiments [3], and the squares are the calculated results with $E=37200 \mathrm{~cm}^{-1}$ and $J=3552 \mathrm{~cm}^{-1}$. 
(a)
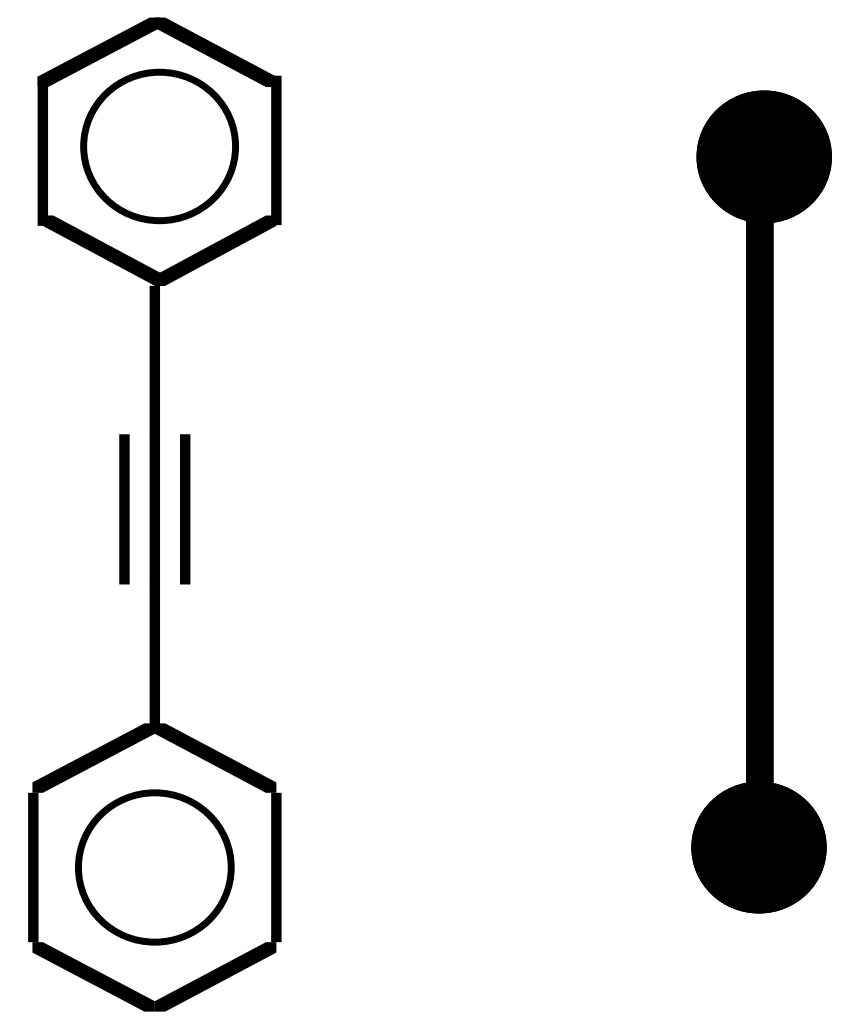
(b)

$d$

D4

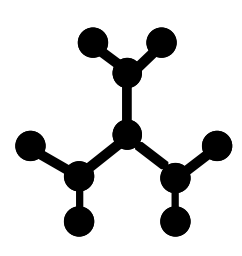

D10

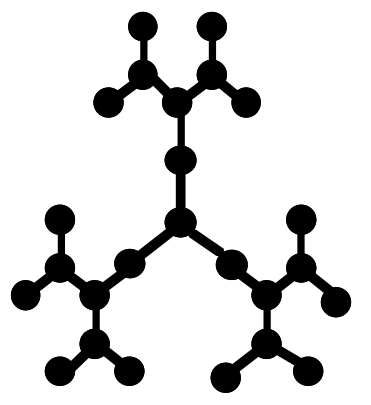

D25

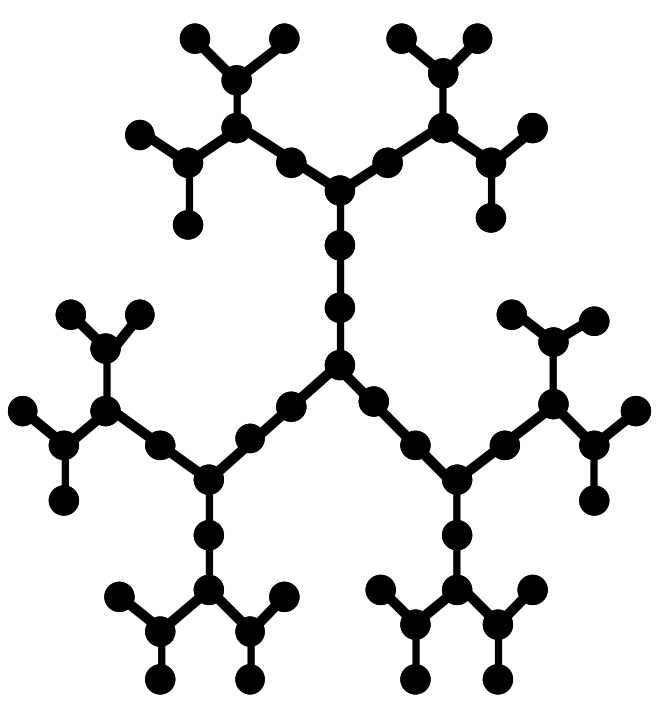

D58

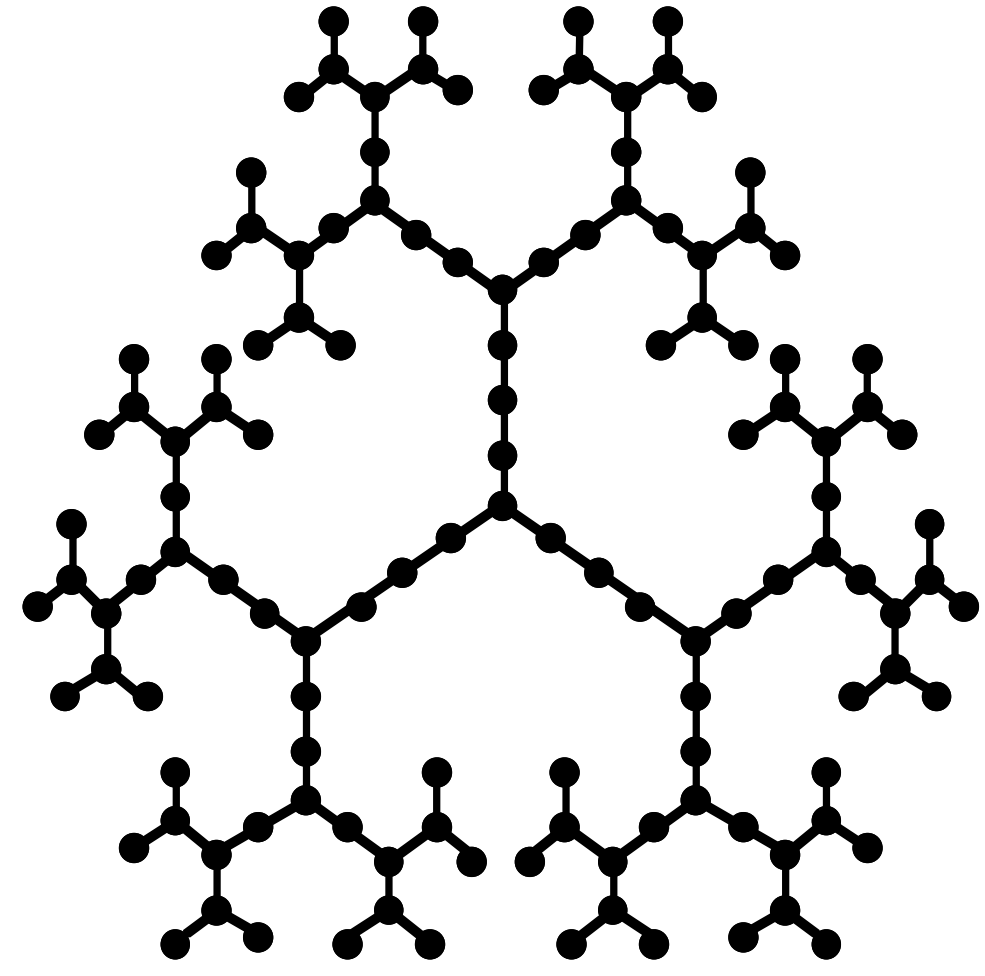

D127 


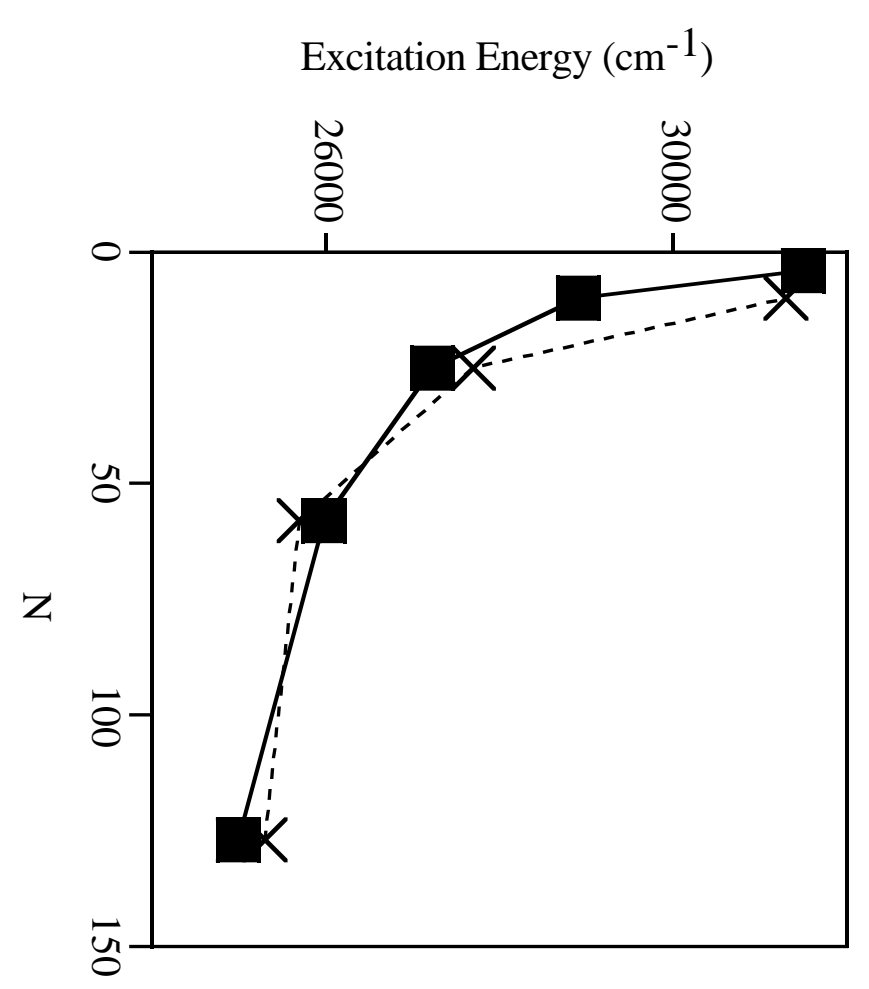

